\title{
Varied EMG responses of the quadriceps muscle during a Wingate anaerobic test
}

\author{
Alexandre Hideki Okano ${ }^{(1)}$, Antonio Carlos de Moraes(1), Antonia Dalla Pria Bankoff(1), Edilson Serpeloni Cyrino(2), \\ Herbert Gustavo Simões ${ }^{(3)}$, Pedro Pezarat-Correia ${ }^{(4)}$ João Francisco Barbieri(1), Cássia Lopes Teodoro ${ }^{(1)}$, \\ Arthur Fernandes Gáspari(1)
}

\begin{abstract}
Introduction: The Wingate Test (WAnT) has been used to evaluate anaerobic performance in cycle ergometer. Objective: The purpose of the present study was to analyze the EMG response of the superficial quadriceps femoris muscles during the Wingate anaerobic test (WAnT). In addition, we investigated the influence of these muscles in performance and fatigue during the WAnT. Methods: For this purpose eight male cyclists performed a 30-s WAnT. Electromyographic amplitude (RMS) and median power frequency (MPF) data of the vastus lateralis (VL), vastus medialis (VM), and rectus femoris (RF) muscles, performance index (mean power, MP) and rate of fatigue (fatigue index, FI) were recorded during the test; and compared along six time points. The correlation between EMG data and performance parameters were made. Results: No significant differences were found in maximal EMG amplitude for all the muscles. The MPF of the VL and VM muscles decreased throughout the test ( $12 \%$ and $13 \%$, respectively), however, the decline for the RF was twice greater (25\%). Correlation among MP and EMG amplitude was observed only for $V L(r=0.83 ; P<0.01)$. There was significant correlation between the FI and the MPF decrease in the RF muscle only $(r=0.78 ; P<0.05)$. Conclusion: During the WAnT, MPF decrease of RF muscle was significantly larger when compared to the VL and VM. The VL muscle electrical activity was positively correlated with MP, suggesting that VL is an accurate predictor of the performance in the WAnT. The MPF decrease of the RF muscle was positively correlated to the FI. These results may be explained by differences in the fiber type composition, biomechanics and architecture properties of these muscles.
\end{abstract}

Keyword: Wingate anaerobic test, Electromyography, Quadriceps Muscle, Fatigue, Neuromuscular.

\section{INTRODUCTION}

The Wingate Test (WAnT) has been widely used to evaluate anaerobic performance in mechanically and electro-magnetically braked cycle ergometer, ${ }^{(1-3)}$ since it is a non-invasive test that is easily applied, validated and with high reproducibility. ${ }^{(1,4,5)}$ The WAnT requires all-out pedaling or arm cranking for 30 seconds against constant load relative to the body mass of the subject. ${ }^{(1,4)}$

An interesting aspect is that the majority of investigations that have used the WAnT as physical performance index have analyzed the energy systems in isolation ${ }^{(6-10)}$ or combined with the use of ergogenic aid..$^{(11-18)}$ Undoubtedly, information produced by these studies has contributed a lot to understanding metabolic aspects and fatigue mechanisms related to high intensity exercise. Nevertheless, it is important to point out that in general in the above studies the analyses were performed by means of blood samples or muscular biopsy. These investigations provided information about the organic system as a whole or only about a specific muscle, respectively. On the other hand, using surface electromyography (EMG), it is possible that the samples be taken simultaneously in different muscles involved in physical effort. During exercise performed on a cycle ergometer, the vastus lateralis (VL), vastus medialis (VM) and rectus femoris (RF) muscles are the major muscles involved in the pedaling. ${ }^{(19,20)}$

In the light of these considerations, several researchers have found a dissociation in the EMG response between the VL, $\mathrm{VM}$ and RF muscles during the exercise of knee extension. ${ }^{(21-25)}$ Regarding the WAnT, there are little studies that have analyzed the EMG response between QF muscles in maximum effort on the cycle ergometer. ${ }^{(26-28)}$

Thus, we must consider the lack of studies analyzing the EMG responses of the QF muscles simultaneously, so the purpose of the present study was to analyze the EMG response of the VL, VM and RF muscles during the WAnT. We hypothesized that differences in the fiber type composition, biomechanics and

Corresponding author: Cássia Lopes Teodoro. Faculdade de Educação Física - FEF/DCE, Universidade Estadual de Campinas - UNICAMP. Caixa Postal 6134. 13.083-970 Campinas - São Paulo - Brasil. E-mail: cassiars@gmail.com

${ }^{1}$ Faculdade de Educação Física, Universidade Estadual de Campinas - UNICAMP, Campinas/SP, Brasil.

Full list of author information is available at the end of the article.

Financial support: nothing to declare

Submission date 01 February 2017; Acceptance date 30 March 2017; Publication date 12 June 2017 
architecture properties of these muscles would induce in a dissociation of EMG signal responses during the WAnT.

\section{METHODS}

\section{Subjects}

Eight male cyclists (age: $26.9 \pm 6.4$ years; body mass: $74.8 \pm 8.7 \mathrm{~kg}$; height: $179.6 \pm 9.4 \mathrm{~cm}$; body mass index: $23.1 \pm 1.4 \mathrm{~kg} / \mathrm{m}^{2}$ ) volunteered to participate in the study. All were fully informed about the procedures and risks of the study. The subjects were familiar with equipment and protocol. Subsequently, they signed an informed consent term. The study was approved by the local University's Research Ethics Committee according to number 427/2003 and performed in accordance with the declaration of Helsinki.

\section{Anthropometry}

Body mass was measured on a model ID-1500 Filizola scale with margin of error of $0.1 \mathrm{~kg}$. Height was obtained on an stadiometer with a margin of error of $0.1 \mathrm{~cm}$. The individuals were measured in their stocking feet and only wearing light clothes.

\section{WAnT}

Ambient temperature and relative humidity were kept constant during tests. The WAnT was conducted according to the widely accepted recommendations for standardization. ${ }^{(4)}$ Initially, they had a four-minute warm up with a 32 watts load at a cadence between 70 and $80 \mathrm{rpm}$. After the warm up there was an interval of two minutes to measure body mass, adjust the seat height and the intensity of effort. A resistance corresponding to $90 \mathrm{~g}^{\mathrm{kg}} \mathrm{gg}^{-1}$ of the body mass was applied. ${ }^{(29)}$ The subjects performed the WAnT for lower-limb that consisted of maximum intensity pedaling continuously for $30 \mathrm{~s}$. The test was conducted on a mechanically braked cycle ergometer (Monark ${ }^{\circledR}$, Brasil) adapted with optical sensors to count cadence (rpm). The subjects were encouraged verbally to pedal as fast as possible during the test. The performance indexes produced peak power (PP); mean power (MP) and fatigue index (FI) were determined by the Wingate Test computerized program (CEFISE ${ }^{\mathrm{TM}}$ ). After termination of the test the subjects performed an active recovery on the cycle ergometer without resistance for three minutes as an attempt to minimize possible side effects induced by their efforts. The subjects were oriented to not do vigorous physical activities nor eat or drink substances with caffeine (coffee, chocolate, mate tea, guarana powder, Coca Cola, Guarana soft drink) or alcoholic beverages during the 24 hours preceding the test to avoid possible interference.

\section{EMG signal acquisition and processing}

For collecting and processing the EMG signals, a model MP150, Biopac System (Inc., Santa Barbara, CA, USA) containing sixteen channels was used. The sample rate established for acquiring the EMG registers was $1000 \mathrm{~Hz}$. Raw EMG signals were bandpass filtered at $20-500 \mathrm{~Hz}$ and stored on computer disk. The common mode rejection of the current system was $>80 \mathrm{~dB}$. Surface muscles of the $\mathrm{QF}$ (RF, VL and VM) of the dominant lower-limb were analyzed. Bipolar surface active electrodes (model TSD 150, BIOPAC System, Inc., Santa Barbara, CA, USA) were placed on each muscle with an inter electrode distance (center-to-center) of $20 \mathrm{~mm}$. Prior to electrode placement, the skin area was shaved, cleaned with alcohol and abraded in order to reduce skin impedance and to ensure electrode adherence. Electrode placement for the muscles followed the standard procedure. ${ }^{(30)}$

To analyze the EMG responses of the VL, VM and RF muscles, the WAnT was broken up into six periods of five seconds intervals. The frequency spectrum of each epoch of EMG data was analyzed using a Fast Fourier transformation. The mean power frequency (MPF) was then determined. Furthermore, the amplitude of the EMG signals represented by the RMS value was determined in all periods of 5 seconds as an average of this time. Both indexes were normalized by the result obtained in the first five seconds of the test. We employed AcqKnowledge 3.8.1 software (Biopac System, Inc., Santa Barbara, CA, USA) to process the EMG signals.

\section{Statistical analysis}

The results obtained were grouped in mean and standard deviation values. Results of the performance variables and EMG signals of the VL, VM and RF muscles obtained during the WAnT were contrasted by analysis of variances (ANOVA) with repeated measures. To locate possible differences, the Post hoc Tukey test was employed. Correlations between EMG signals obtained from the VL, VM and RF muscles and the physical performance indexes (MP) in the WAnT were established by Pearson correlation. The level of significance adopted for all the analysis was $5 \%$.

\section{RESULTS}

Figure 1 shows the power and EMG signals responses of the VL, VM and RF muscles during the WAnT.

In relation to performance (Figure 1a), there was a time (period) effect during the WanT $(P<0.05)$ indicating that the power suffered significant alterations between the periods in WAnT.

Means and standard deviations of amplitude (RMS) of the EMG signals of the VL, VM and RF muscles every five seconds during WanT are shown in Figure 1b. Regarding amplitude of the RMS, no time effect during the WAnT was verified for all the muscles $(P>0.05)$. Concerning the MPF (Figure 1c), in all muscles investigated a time effect during WanT with significant reductions $(P<0.05)$ during the course of the test was verified, the RF differed statistically from the VL and VM muscles and presented greater reduction of the MPF over the 30 s period. 


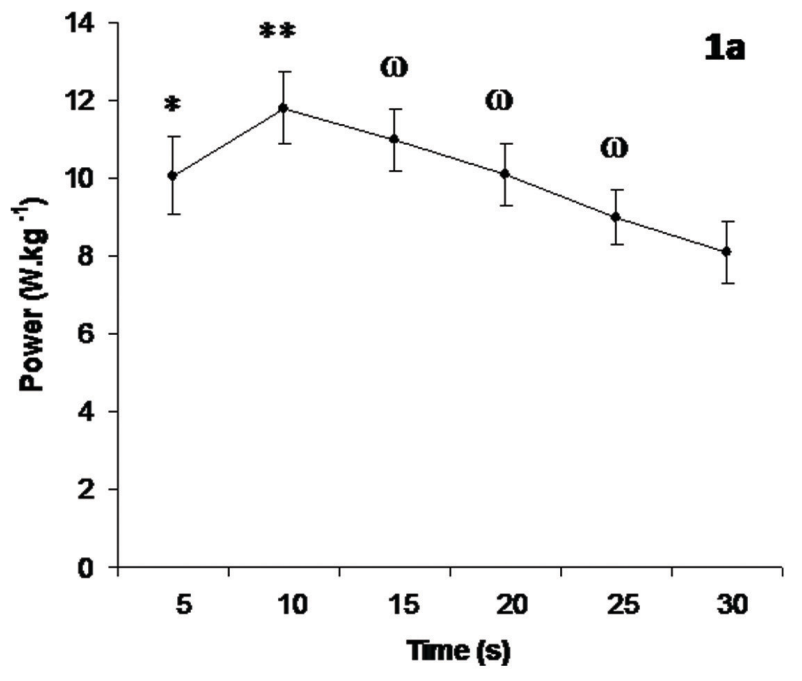

Figure 1a. Peak power. "*” Significant differences from periods 10s, 25s, and 30s $(P<0.01){ }^{* *}$ Significant differences from periods $20 \mathrm{~s}, 25 \mathrm{~s}$, and $30 \mathrm{~s}$ $(P<0.01)$. " $\omega$ " Significant differences from subsequent periods of five seconds intervals $(P<0.01)$.

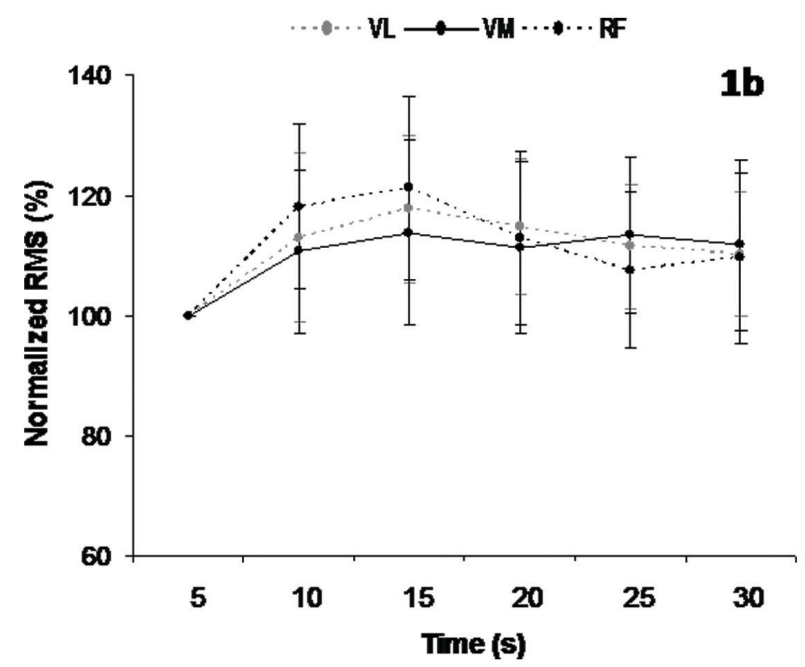

Figure 1b. Electromyographic amplitude (RMS).

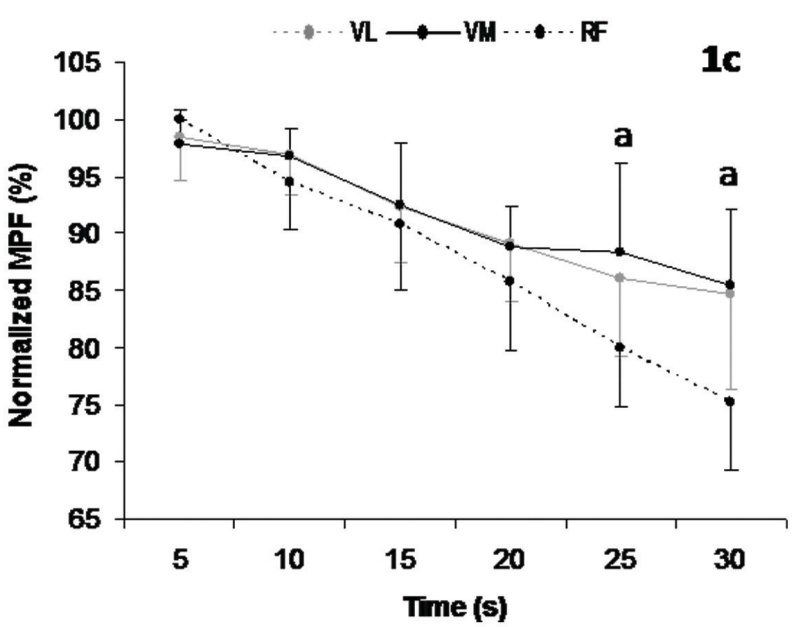

Figure 1c. Median power frequency (MPF) during the Wingate anaerobic test. "a" Significant differences from the vastus lateralis and vastus medialis $(P<0.05)$.
Table 1 shows the results of correlation including information related to the muscular activation of the VL, VM and RF muscles and the MP obtained in the WAnT.

It was found that just activation of the VL muscle were correlated $(P<0.01)$ to MP in the WAnT.

In Table 2, there can be seen correlations obtained between the $\mathrm{FI}$ and the MPF decrease during the WAnT.

There was significant correlation between the $\mathrm{FI}$ and the MPF decrease in the RF muscle only.

\section{DISCUSSION}

As main find of this research, a dissociation in the responses of the QF muscles was observed. Regarding initial values, the VL and VM muscles presented reductions of $12-13 \%$ during the test. On the other hand, the drop of the RF muscle was $25 \%$. Findings of the present study are similar to those of other researchers $^{(21,22,31-33)}$ who found a dissociation in the EMG responses of the $\mathrm{VL}, \mathrm{VM}$ and $\mathrm{RF}$ muscles in the face of the fatigue protocols in the exercise of knee extension. The same results was observed during the protocol identifying the EMG fatigue threshold in cycle ergometer. ${ }^{(34)}$ It was seen that the intensity corresponding to EMG fatigue threshold for the RF muscle was around $1-23 \%$ less than that obtained by the VL. One aspect that could explain this result is the difference in the proportion of fiber type between muscles. In a study of six cadavers, it was found that the RF is constituted predominantly by type II fibers when compared to the VL and VM muscles. ${ }^{(35,36)}$ The RF presents a specific characteristic in relation to the rest of the muscles that compose the QF, possible because it is a bi-articulated muscle, it is responsible for extending the knees and flexing the hips while the VL and VM muscles are mono-articulated and promote only extension of the knees. $^{(19,20,37)}$ In relation to the VL and VM muscles, although both have similar anatomic properties, the characteristics regarding fiber type composition show differences. The VL is constituted of a greater proportion of type II fibers in

Table 1. Correlation among mean power and electromyographic amplitude of quadriceps muscles during Wingate anaerobic test.

\begin{tabular}{ccc}
\hline & $\boldsymbol{r}$ & $\boldsymbol{P}$ \\
\hline MP $\times$ RMS-VL & 0.83 & 0.01 \\
MP $\times$ RMS-VM & 0.12 & 0.78 \\
MP $\times$ RMS-RF & 0.40 & 0.33 \\
\hline
\end{tabular}

Mean power (MP), electromyographic amplitude (RMS), vastus lateralis (VL), vastus medialis (VM) and rectus femoris (RF).

Table 2. Correlation among variables during Wingate anaerobic test.

\begin{tabular}{ccc}
\hline & $\boldsymbol{r}$ & $\boldsymbol{P}$ \\
\hline FI $x$ VL & 0.31 & 0.44 \\
FI XVM & 0.56 & 0.15 \\
FI x RF & 0.78 & 0.02 \\
\hline
\end{tabular}

Fatigue index (FI), vastus lateralis (VL), vastus medialis (VM) and rectus femoris (RF) 
relation to the VM. ${ }^{(35,38,39)}$ In addition, the VL presents a greater cross-sectional area than the VM, resulting in greater absolute muscular tension and intra-muscular pressure. ${ }^{(20,33)}$ In situations where intra-muscular pressure is increased, blood flow for the active muscle is diminished, which contributes to the instauration of an anaerobic condition. ${ }^{(40)}$ Based on these facts, the expected results would be differentiated responses of the two muscles in the fatigue condition. However, in the present study the results do not support the hypothesis raised. In both muscles, reduction of the MPF was similar (12-13\%); one of the possible explanations for these results can be linked to crosstalk. Crosstalk refers to interference of one signal by others that emanate from adjacent muscles. ${ }^{(41,42)}$ In this sense, hypothetically, we would have interference of the synergist muscles (RF and Vastus Intermedius) in picking up signals. It is important to consider that the VM muscle is located at the level of the tendon of the RF and vastus intermedius muscles. Therefore, in this case, the crosstalk would be a problem above all of the VL muscle. This contribution of the synergist muscles signals could result in underestimated fatigue patterns that could lead us to erroneous interpretations.

Concerning the influence of the muscles on performance (Table 1), a significant correlation was found only between VL muscle and MP. A possible explanation for that is related to morphological aspects of the muscles. The cross-sectional area of the $\mathrm{VL}$ is greater than the VM and RF muscles. ${ }^{(20,33)}$ Hence, the VL muscle shows greater capacity for tension than the rest of the muscles. ${ }^{(20)}$

Among the muscles analyzed in this present study, only the RF was correlated with the FI presented in WAnT (Table 2). The RF has the largest proportion of type II fibers $(70.5 \%)^{(33)}$ and as suggested by Hannukainen ${ }^{(43)}$ muscles with large type two fibers receive lass blood flow, being more susceptible to fatigue. Results indicate that the subjects who showed the greatest capacity for maintaining the MPF of the RF apparently are those who probably suffered less fatigue during the effort.

\section{CONCLUSION}

In summary, the amplitude response of the EMG signals (RMS) of the muscles investigated was similar during the WAnT. Regarding the MPF, the magnitude of the decrease of RF muscle was significantly larger when compared to the VL and VM. The VL muscle contributes the most to better MP performance in the WAnT. In relation to the fatigue instauration, the MPF decrease of the RF muscle was correlated to the drop in performance. It should be pointed out that in the present study only the QF muscles were analyzed. Nevertheless, other muscles have effective participation in the action of pedaling. In the light of all this, there is a need for new investigations involving other muscle groups in order to verify the participation as well as the level of fatigue of the muscles during the WAnT.

\section{AUTHOR'S CONTRIBUTION}

AHO, ADPB, PPC: conceived and designed the experiments; AHO, ADPB, ESC, HGS: acquisition of data; ESC, JFB, CLT, AFG: analyzed and interpreted of data; AHO, HGS, PPC, JFB, CLT, AFG: review the literature and wrote; ACM: conceived and designed the experiments, final approved of the version to be published.

\section{CONFLICTS OF INTEREST}

Nothing to declare.

\section{AUTHOR DETAILS}

${ }^{2}$ Centro de Educação Física e Esportes, Universidade Estadual de Londrina UEL, Londrina/PR, Brasil.

${ }^{3}$ Faculdade de Educação Física, Universidade Católica de Brasília - UCB, Brasília/DF, Brasil

${ }^{4}$ Faculty of Human Kinetics, Technical University of Lisbon - UTL, Lisbon, Portugal

\section{REFERENCES}

1. Bar-Or O. The Wingate anaerobic test. An update on methodology, reliability and validity. Sports medicine. 1987;4(6):381-94.

2. Bulbulian R, Jeong JW, Murphy M. Comparison of anaerobic components of the Wingate and Critical Power tests in males and females. Medicine and science in sports and exercise. 1996;28(10):1336-41.

3. Micklewright D, Alkhatib A, Beneke R. Mechanically versus electromagnetically braked cycle ergometer: performance and energy cost of the Wingate Anaerobic Test. European journal of applied physiology. 2006;96(6):748-51.

4. Inbar O, Bar-Or O, Skinner J. The Wingate anaerobic test: development, characteristics, and application. Champaign (IL): Human Kinetics. 1996.

5. Vandewalle H, Peres G, Monod H. Standard anaerobic exercise tests. Sports medicine. 1987;4(4):268-89.

6. Beneke R, Pollmann C, Bleif I, Leithauser RM, Hutler M. How anaerobic is the Wingate Anaerobic Test for humans? European journal of applied physiology. 2002;87(4-5):388-92.

7. Bogdanis GC, Nevill ME, Boobis LH, Lakomy HK, Nevill AM. Recovery of power output and muscle metabolites following $30 \mathrm{~s}$ of maximal sprint cycling in man. The Journal of physiology. 1995;482 (Pt 2):467-80.

8. Vincent S, Berthon P, Zouhal H, Moussa E, Catheline M, Bentue-Ferrer $D$, et al. Plasma glucose, insulin and catecholamine responses to a Wingate test in physically active women and men. European journal of applied physiology. 2004;91(1):15-21.

9. Weinstein Y, Bediz C, Dotan R, Falk B. Reliability of peak-lactate, heart rate, and plasma volume following the Wingate test. Medicine and science in sports and exercise. 1998;30(9):1456-60.

10. Lovell D, Kerr A, Wiegand A, Solomon C, Harvey L, McLellan C. The contribution of energy systems during the upper body Wingate anaerobic test. Applied physiology, nutrition, and metabolism = Physiologie appliquee, nutrition et metabolisme. 2013;38(2):216-9.

11. Bell DG, Jacobs I, Ellerington K. Effect of caffeine and ephedrine ingestion on anaerobic exercise performance. Medicine and science in sports and exercise. 2001;33(8):1399-403.

12. Casey A, Constantin-Teodosiu D, Howell S, Hultman E, Greenhaff PL. Creatine ingestion favorably affects performance and muscle metabolism during maximal exercise in humans. The American journal of physiology. 1996;271(1 Pt 1):E31-7.

13. Collomp K, Le Panse B, Portier $H$, Lecoq AM, Jaffre $C$, Beaupied $H$, et al. Effects of acute salbutamol intake during a Wingate test. International journal of sports medicine. 2005;26(7):513-7.

14. Engels HJ, Kolokouri I, Cieslak TJ, 2nd, Wirth JC. Effects of ginseng supplementation on supramaximal exercise performance and short-term recovery. Journal of strength and conditioning research. 2001;15(3):290-5. 
15. Greer F, McLean C, Graham TE. Caffeine, performance, and metabolism during repeated Wingate exercise tests. Journal of applied physiology. 1998;85(4):1502-8.

16. Kocak S, Karli U. Effects of high dose oral creatine supplementation on anaerobic capacity of elite wrestlers. The Journal of sports medicine and physical fitness. 2003;43(4):488-92

17. Kreider RB, Melton C, Greenwood M, Rasmussen C, Lundberg J, Earnest C, et al. Effects of oral D-ribose supplementation on anaerobic capacity and selected metabolic markers in healthy males. International journal of sport nutrition and exercise metabolism. 2003;13(1):76-86.

18. Fujii N, Tsuchiya S, Tsuji B, Watanabe K, Sasaki Y, Nishiyasu T. Effect of voluntary hypocapnic hyperventilation on the metabolic response during Wingate anaerobic test. European journal of applied physiology. 2015;115(9):1967-74

19. Jorge M, Hull ML. Analysis of EMG measurements during bicycle pedalling. Journal of biomechanics. 1986;19(9):683-94.

20. Driss $\mathrm{T}$, Vandewalle $\mathrm{H}$. The measurement of maximal (anaerobic) power output on a cycle ergometer: a critical review. BioMed research international. 2013;2013:589361.

21. Ebenbichler G, Kollmitzer J, Quittan M, UhI F, Kirtley C, Fialka V. EMG fatigue patterns accompanying isometric fatiguing knee-extensions are different in mono- and bi-articular muscles. Electroencephalography and clinical neurophysiology. 1998;109(3):256-62.

22. Kouzaki M, Shinohara M, Fukunaga T. Non-uniform mechanical activity of quadriceps muscle during fatigue by repeated maximal voluntary contraction in humans. European journal of applied physiology and occupational physiology. 1999;80(1):9-15

23. Vaz MA, Zhang YT, Herzog W, Guimaraes AC, Maclntosh BR. The behavior of rectus femoris and vastus lateralis during fatigue and recovery: an electromyographic and vibromyographic study. Electromyography and clinical neurophysiology. 1996;36(4):221-30.

24. Wretling ML, Henriksson-Larsen K, Gerdle B. Inter-relationship between muscle morphology, mechanical output and electromyographic activity during fatiguing dynamic knee-extensions in untrained females. European journal of applied physiology and occupational physiology. 1997;76(6):483-90.

25. Akima $\mathrm{H}$, Saito A. Activation of quadriceps femoris including vastus intermedius during fatiguing dynamic knee extensions. European journal of applied physiology. 2013;113(11):2829-40.

26. Hunter AM, St Clair Gibson A, Lambert MI, Nobbs L, Noakes TD. Effects of supramaximal exercise on the electromyographic signal. British journal of sports medicine. 2003;37(4):296-9.

27. Vandewalle H, Maton B, Le Bozec S, Guerenbourg G. An electromyographic study of an all-out exercise on a cycle ergometer. Archives internationales de physiologie, de biochimie et de biophysique. 1991;99(1):89-93.

28. Souissi H, Chtourou H, Chaouachi A, Chamari K, Souissi N, Amri M. Time-ofDay Effects on EMG Parameters During the Wingate Test in Boys. Journal of sports science \& medicine. 2012;11(3):380-6.

29. Okano AH, Altimari LR, Moraes AC, Teixeira O, Ravagnani FC, Coelho $C F$, et al. Comparison between individual anaerobic threshold and ventilatory threshold determined in cyclists. Medicine \& Science in Sports \& Exercise. 2003;35(5):S118.
30. Hermens HJ, Freriks B, Disselhorst-Klug C, Rau G. Development of recommendations for SEMG sensors and sensor placement procedures. Journal of electromyography and kinesiology: official journal of the International Society of Electrophysiological Kinesiology. 2000;10(5):36174.

31. Kinugasa R, Hayashi S, lino N, Tamura M, Oouchi T, HORI A. Recruitment pattern of quadriceps femoris muscles during repetitive knee extension exercise by muscle functional MRI. Journal of Physical Exercise and Sports Science, Tokyo. 2002;8:1-6.

32. Tesch PA, Dudley GA, Duvoisin MR, Hather BM, Harris RT. Force and EMG signal patterns during repeated bouts of concentric or eccentric muscle actions. Acta physiologica Scandinavica. 1990;138(3):263-71.

33. Wickiewicz TL, Roy RR, Powell PL, Edgerton VR. Muscle architecture of the human lower limb. Clinical orthopaedics and related research. 1983(179):275-83.

34. Housh TJ, deVries HA, Johnson GO, Housh DJ, Evans SA, Stout JR, et al. Electromyographic fatigue thresholds of the superficial muscles of the quadriceps femoris. European journal of applied physiology and occupational physiology. 1995;71(2-3):131-6.

35. Johnson MA, Sideri G, Weightman D, Appleton D. A comparison of fibre size, fibre type constitution and spatial fibre type distribution in normal human muscle and in muscle from cases of spinal muscular atrophy and from other neuromuscular disorders. Journal of the neurological sciences. 1973;20(4):345-61.

36. Aagaard P, Andersen JL, Dyhre-Poulsen P, Leffers AM, Wagner A, Magnusson SP, et al. A mechanism for increased contractile strength of human pennate muscle in response to strength training: changes in muscle architecture. The Journal of physiology. 2001;534(Pt. 2):613-23.

37. Bressel E. Biomechanical Basis of Human Movement. Medicine \& Science in Sports \& Exercise. 2003;35(10):1795.

38. Edgerton VR, Smith JL, Simpson DR. Muscle fibre type populations of human leg muscles. The Histochemical journal. 1975;7(3):259-66.

39. Watanabe K, Akima H. Neuromuscular activation of vastus intermedius muscle during fatiguing exercise. Journal of electromyography and kinesiology: official journal of the International Society of Electrophysiological Kinesiology. 2010;20(4):661-6.

40. Grabiner MD, Koh TJ, Miller GF. Fatigue rates of vastus medialis oblique and vastus lateralis during static and dynamic knee extension. Journal of orthopaedic research: official publication of the Orthopaedic Research Society. 1991;9(3):391-7.

41. Basmajian JV, De Luca C. Muscles alive. Muscles alive: their functions revealed by electromyography. 1985;278:126.

42. McCool P, Fraser GD, Chan AD, Petropoulakis L, Soraghan JJ. Identification of contaminant type in surface electromyography (EMG) signals. IEEE transactions on neural systems and rehabilitation engineering: a publication of the IEEE Engineering in Medicine and Biology Society. 2014;22(4):774-83.

43. Hannukainen JC, Nuutila P, Kaprio J, Heinonen OJ, Kujala UM, Janatuinen T, et al. Relationship between local perfusion and FFA uptake in human skeletal muscle-no effect of increased physical activity and aerobic fitness. Journal of applied physiology. 2006;101(5):1303-11. 